\title{
Fuzzy choice functions, consistency, and sequential fuzzy choice
}

\author{
José Carlos R. Alcantud, ${ }^{1}$ S. Díaz ${ }^{2}$ \\ ${ }^{1}$ Facultad de Economía y Empresa and IME, University of Salamanca, 37007 Salamanca, Spain \\ ${ }^{2}$ Dept. of Statistics and O. R., University of Oviedo, Calvo Sotelo s/n, 33007 Oviedo, Spain
}

\begin{abstract}
In the setting of fuzzy choice functions (Georgescu [6]), we explore the relationships among known and new consistency axioms. Then we define the notion of sequential application of fuzzy choice functions, and investigate its normative implications. The Fuzzy Arrow Axiom is preserved by this sequential procedure, which ensures that full-rationality is preserved too.
\end{abstract}

Keywords: Choice function, Fuzzy choice function, Fuzzy Arrow Axiom.

\section{Introduction}

In the act of choice, rationality is a central issue that has raised many controversial analysis. There is a trend in the literature that considers choice as rational only when derives from well-behaved preferences. Transitivity of preference is the paradigmatic rational principle to obey, and it is assumed by many descriptive theories of decision making. However experimental evidence contradicts this position, as recognized as soon as in May [9] and Tversky [12].

Therefore other authors attempted to study the object of choice-formally, choice functions- and discuss which possible rationality properties could be tested without any reference to an underlying preference. As soon as in Uzawa [13] the term "rational choice function" means a choice function satisfying axioms of rational behavior. Nevertheless the idea that these axioms can be used to test the model by optimization of nice preferences with the observables (the actual choices) is central in the analysis of economic or abstract decisions (cf., Arrow [1] and Sen [10] among others).

If we recognize that social phenomena involve intrinsically vague concepts then we can perform more extensive studies in the lines highlighted above. In this work we are interested in fuzzy choice functions as defined in Georgescu [6].

We explore the relationships among known and new consistency axioms for this setting. Then we define the new concept of sequential fuzzy choice functions, and explore its normative implications. Our main findings are two when the minimum tnorm is assumed. Firstly, a foremost consistency axiom known as Fuzzy Arrow Axiom is preserved by this sequential procedure. This implies that the same is true for full-rationality (i.e., for the existence of a well-behaved fuzzy relation that Grationalizes the fuzzy choice).

Secondly, when the sequential application of G-rational fuzzy choice functions produces a Grational fuzzy choice function, the fuzzy preference that G-rationalizes their sequential application is identified.

This paper is structured as follows. In Section 2 we recall some preliminary definitions and concepts from the crisp literature. In Section 3 we introduce the fuzzy set theory and discuss the connection between some consistency conditions in this context. In Section 4 we present the results obtained for sequential choices. We conclude in Section 5.

\section{The Crisp Case}

The remote antecedent of our investigation is the following model of abstract choice.

The collection of all situations that a decisionmaker can conceivably face are represented by $\mathcal{B}$, a nonempty domain of nonempty subsets of $X$. That is, if $\mathcal{P}(X)$ denotes the set of all subsets of $X$ then we require $\emptyset \neq \mathcal{B} \subseteq \mathcal{P}(X)$ and $S \neq \emptyset$ for all $S \in \mathcal{B}$. For convenience sometimes we drop brackets, e.g., the set $\{x\}$ can be represented as $x$.

Choices (among the subsets in $\mathcal{B}$ ) are captured by the following notion:

Definition $1 A$ decisive choice correspondence on $(X, \mathcal{B})$ is a map $C: \mathcal{B} \rightarrow \mathcal{P}(X)$ such that $C(S) \subseteq S$ and $C(S) \neq \emptyset$ for all $S \in \mathcal{B}$.

Henceforth we simply refer to decisive choice correspondences as choice functions. They attach with each feasible set in the domain $\mathcal{B}$, the set of alternatives that are either potentially or actually chosen by the decision-maker.

Particular specifications of this abstract model call for suitably adapted terms. For example in Economics, demand theory is concerned with demand functions or demand correspondences, whose domain is constituted by budget sets.

\subsection{Consistency properties}

A good deal of the literature on choice functions is devoted to study properties of choice that embody 
rationality in various forms and their relationships. We proceed to list some rationality or consistency properties of choice that we are interested in.

The strongest version of the consistency axioms that we define is the crisp Arrow Axiom (AA), which demands that for any $S, T \in \mathcal{B}$, the following implication holds:

$S \subseteq T \Rightarrow$ either $S \cap C(T)=\emptyset$ or $S \cap C(T)=C(S)$.

Other weaker properties used to capture rationality in the context of crisp choice functions follow:

Definition 2 The choice function $C$ satisfies:

- The Chernoff condition, also $\mathbf{C H}$, if for any $S, T \in \mathcal{B}$ such that $S \subseteq T$ we have

$$
C(T) \cap S \subseteq C(S) .
$$

- The Superset property, also SUP, if for all $S, T \in \mathcal{B}$ such that $S \subseteq T$ it is the case that

$$
C(T) \subseteq C(S) \Rightarrow C(T)=C(S) .
$$

- Property $\gamma$ (cf., Sen [10]), also $\gamma$, if for any collection $\left\{M_{i}\right\}_{i \in I}$ of subsets of $\mathcal{B}$ the following holds true:

$$
x \in C\left(M_{i}\right) \text { for all } i \in I \text { entails } x \in C\left(\cup_{i \in I} M_{i}\right) \text {. }
$$

This property is stronger than the Concordance property which only establishes that $C(S) \cap C(T) \subseteq C(S \cup T)$ throughout.

- The Binariness property, also $\mathbf{B}$, if for any $S \in \mathcal{B}$ we have: $x \in S$ and $x \in C(x, y)$ for all $y \in S$ implies $x \in C(S)$.

\subsection{Relationships among consistency properties}

In the crisp case there are implications among the properties in Subsection 2.1, which are represented by Figure 1. Neither of them can be reversed. Moreover, there is no connection among the properties on the right, except for the ones shown between Property $\gamma$ and Binariness and Property $\gamma$ and Concordance.

$$
\text { Arrow Axiom } \Rightarrow\left\{\begin{array}{c}
\text { Chernoff condition } \\
\text { Superset property } \\
\text { Property } \gamma \Rightarrow \text { Binariness } \\
\Downarrow \\
\text { Concordance }
\end{array}\right.
$$

Figure 1: Implications among consistency properties of crisp choice functions.

The relationships among the consistency properties mentioned above and the possibility that the choice function can be rationalized through some specific procedure constitute another important branch in choice theory, especially in revealed preference theory. These relationships and characterizations rely on the structure of the domain of subsets for which choices are defined or known. Particularly, we may be bound by the following constraints:

Definition 3 Condition $H$ is satisfied if $\mathcal{B}$ contains all non-empty finite subset of $X$. Condition $W H$ is satisfied if $\mathcal{B}$ contains at least all the pairs and triplets of alternatives.

To grasp the importance of assumption $\mathrm{H}$ we recall that already Arrow [1] emphasized in his insightful analysis on the theory of consumer's demand: "the demand-function point of view would be greatly simplified if the range over which the choice functions are considered to be determined is broadened to include all finite sets" and also: "requiring the choice functions to be defined for finite sets is thoroughly consistent with the intuitive arguments underlying revealed preference. It should also be observed that any hope of using experimental methods for studying preference will require inferring from choices on finite sets to choices on infinite ones." Even under assumption WH, he shows among other issues that Samuelson's weak axiom of revealed preference is equivalent to the strong axiom of revealed preference and to Arrow's Axiom. And that they are equivalent to the property that the choice function is rationalizable in the most satisfactory manner: there exists a complete and transitive relation $Q$ such that for each $S \in \mathcal{B}, C(S)$ is the set of maximizers of $R$ in $S$, this is,

$$
C(S)=\{x \in S \mid(x, y) \in R \text { for all } y \in S\}
$$

\section{The Fuzzy Case: a Study in Consistency}

We now move forward to the fuzzy context. We begin with the analysis of the issues that Section 2 motivates. The main concept that we need to establish is the following (cf., Georgescu [6, Definition $5.13])$ :

Definition 4 Let $X$ be a non-empty set and $\mathcal{B}$ a non empty set of non-zero fuzzy subsets of $X$.

A fuzzy choice function on $(X, \mathcal{B})$ is a mapping $C: \mathcal{B} \rightarrow \mathcal{F}(X)$ such that for each $S \in \mathcal{B}, C(S)$ is non-zero and $C(S) \subseteq S$, i.e., $C(S)(x) \leq S(x)$ for all $x \in X$.

$S(x)$ is called the availability degree of the alternative $x$ in $S$ or if $S$ represents a vague criterion, it captures the degree to which $x$ verifies the criterion. Definition 4 includes the class of fuzzy choice functions originally studied by Banerjee [2].

In order to define consistency properties of fuzzy choice functions, we first need to recall the concepts 
that permit to use intersection, union, implications and inclusions in the fuzzy context.

The intersection of fuzzy sets is usually defined by the minimum t-norm, and we follow this tradition. A t-norm is a binary operation $*$ on $[0,1]$ that is commutative, associative, monotone and has 1 as neutral element. The most popular t-norm is the minimum. Thus given two fuzzy sets $A$ and $B$, we set $(A \cap B)(x)=\min (A(x), B(x))$ for all $x \in X$. The union of these two sets is defined using the maximum: $(A \cup B)(x)=\max (A(x), B(x))$ for all $x \in X$. T-norms are used to model conjunction in fuzzy logic too.

Given a t-norm $*$, the (residual) implication associated to $*$ is the relation $\rightarrow_{*}$ defined as

$$
x \rightarrow_{*} y=\sup \{z \in[0,1] \mid x * z \leq y\} .
$$

The biresiduum of $*$ is defined by

$$
x \leftrightarrow_{*} y=\min \left(x \rightarrow_{*} y, y \rightarrow_{*} x\right) .
$$

If no confusion is possible, we will just write $\rightarrow$ and $\leftrightarrow$. For the minimum t-norm,

$$
x \rightarrow y= \begin{cases}1 & , \text { if } x \leq y \\ y & , \text { otherwise }\end{cases}
$$

and

$$
x \leftrightarrow y= \begin{cases}1 & , \text { if } x=y \\ \min \{x, y\} & , \text { otherwise }\end{cases}
$$

Given a left-continuous t-norm *, the degree of inclusion of the set $S$ in the set $T$ is

$$
I(S, T)=\bigwedge_{x \in X}(S(x) \rightarrow T(x)),
$$

and the degree of equality between $S$ and $T$ is

$$
E(S, T)=\min (I(S, T), I(T, S)) .
$$

For the minimum t-norm, the expressions above boil down to

$$
I(S, T)=\bigwedge\{T(x): x \in X, S(x)>T(x)\}
$$

and

$$
E(S, T)=\bigwedge\{S(x) \wedge T(x): x \in X, S(x) \neq T(x)\}
$$

A property of the degree of inclusion that facilitates the proofs of Lemma 2 and Theorem 1 below is the following: if $T_{1} \subseteq T_{2}$ and $S$ are three fuzzy subsets of a set $X$, then

$$
E\left(S, T_{1}\right) \leq I\left(S, T_{1}\right) \leq I\left(S, T_{2}\right) .
$$

For a more extensive reference about properties of the usual operations with fuzzy sets the reader may consult $[3,4]$.

\subsection{Consistency of fuzzy choice functions: some new axioms}

Let $X$ be a non-empty set and $\mathcal{B}$ a non empty set of non-zero fuzzy subsets of $X$. We proceed to define some known and new axioms of consistency for $C$,

a fuzzy choice function on $(X, \mathcal{B})$.

Georgescu [6, subsection 6.5] extended the Arrow Axiom to the context of fuzzy choice functions as follows:

Definition 5 The fuzzy choice function $C$ satisfies the Fuzzy Arrow Axiom (FAA) if for any $S, T \in$ $\mathcal{B}$ and $x \in X$ we have

$$
I(S, T) * S(x) * C(T)(x) \leq E(S \cap C(T), C(S)) .
$$

Analogously, we now propose to extend the consistency properties of choice functions in Definition 2 , in the following terms:

Definition 6 The fuzzy choice function $C$ satisfies:

- The fuzzy Chernoff condition, also $\mathbf{F C H}$, if for any $S, T \in \mathcal{B}$,

$$
I(S, T) \leq I(C(T) \cap S, C(S)) .
$$

- The fuzzy Superset property, also FSUP, if for all $S, T \in \mathcal{B}$ it holds that

$$
I(S, T) * I(C(T), C(S)) \leq E(C(T), C(S)) .
$$

- The fuzzy Concordance property, also FC, if for any $S, T \in \mathcal{B}$ such that $S \cup T \in \mathcal{B}$, the following holds true for all $x \in X$ :

$$
C(S)(x) * C(T)(x) \leq C(S \cup T)(x) .
$$

- The fuzzy Property $\gamma$ (cf., Sen [10]), also $\mathbf{F} \gamma$, if for any collection $\left\{M_{i}\right\}_{i \in I}$ of subsets of $\mathcal{B}$ such that $\cup_{i \in I} M_{i} \in \mathcal{B}$ the following holds true for all $x \in X$ :

$$
C\left(M_{1}\right)(x) * \cdots * C\left(M_{n}\right)(x) \leq C\left(\cup_{i \in I} M_{i}\right)(x) .
$$

- The fuzzy Binariness property, also FB if for any $S \in \mathcal{B}$ we have:

$$
\begin{gathered}
S(x) * \bigwedge_{y \in X}(S(y) \rightarrow C(\{x, y\})(x)) \leq C(S)(x), \\
\text { for all } x \in X .
\end{gathered}
$$

This property is called Fү2 in Georgescu [6, p. 132].

Remark 1 The following two remarks concerning other fuzzifications of the Chernoff condition are in order.

1. Georgescu [6, subsection 6.5] defines a weaker version of the fuzzy Chernoff condition, that we abbreviate as $\mathbf{F C H}$. In her statement $\mathrm{FCH}^{*}$ establishes that for any $S, T \in \mathcal{B}$,

$I(S, T) * S(x) * C(T)(x) \leq I(C(T) \cap S, C(S))$.

It is clear that $\mathrm{FCH}$ implies $\mathrm{FCH}^{*}$. We return to this property later on in this paper. 
2. Georgescu [6] also defines a dual version of $\mathrm{FCH}^{*}$ that coupled with $\mathrm{FCH}^{*}$ is trivially equivalent to FAA. Similarly one could consider a fuzzy dual Chernoff condition establishing that for any $S, T \in \mathcal{B}$,

$$
I(S, T) \leq I(C(S), C(T) \cap S) .
$$

However this property seems useless for normative analyses because it is not even implied by FAA, as Counterexample 1 below proves.

As in the crisp analysis, in order to study the main features of these new ideas we need to impose properties on the structure of $\mathcal{B}$. Here we follow the direction of Definition 3.

For the purpose of extending condition $\mathrm{H}$ to the fuzzy case, one can consider two options. The first one was proposed by Georgescu [6, subsection 5.2]:

\section{Definition 7 The fuzzy choice function $C$ satisfies}

- Condition H1 if all $S$ and $C(S)$ are normal fuzzy sets, i.e., if for all $S$ there exists an element $x \in X$ such that $C(S)(x)=1$.

- Condition H2 if $\mathcal{B}$ contains all the non-empty crisp finite subsets of $X$, i.e., if $\mathcal{B}$ contains $f[X]=\left\{\left[x_{1}, \ldots, x_{n}\right]: n \geq 1, x_{1}, \ldots, x_{n} \in X\right\}$.

The following alternative possibility was proposed by Martinetti et al. [8] and is less restrictive:

\section{Definition 8 The fuzzy choice function $C$ satisfies}

- Condition WH1 if all $S \in \mathcal{B}$, there exists an element $x \in X$ such that $S(x)>0$ and $C(S)(x)=S(x)$.

- Condition WH2 if $\mathcal{B}$ contains $\{x\},\{x, y\}$ and $\{x, y, z\}$ for all $x, y, z \in X$.

Provided that H1 and H2 hold true, the Fuzzy Arrow Axiom plays a crucial role in revealed preference analysis. Under the minimum t-norm, Georgescu [6, Theorem 6.40] shows the equivalence of FAA with the fuzzy versions of the two most relevant axioms in that contex, to wit, the Weak and the Strong Axioms of Revealed Preference (i.e., WARP and SARP respectively). And most importantly, it establishes the equivalence of any of these axioms with the property that the choice function is full rational.

Recall that the fuzzy choice function $C$ on $(X, \mathcal{B})$ is G-rational if there exists a fuzzy preference relation $Q$ on $X$ such that for any $S \in \mathcal{B}, C(S)=$ $G(S, Q)$. In this regard, $G(S, Q)$ generalizes to fuzzy choice functions the notion of $Q$-greatest elements according to the expression: for each $x \in X$,

$$
G(S, Q)(x)=S(x) * \bigwedge_{y \in X}(S(y) \rightarrow Q(x, y))
$$

In a more restricted sense, $C$ is full-rational if there exists a fuzzy relation $Q$ on $X$ that is reflexive, $*$-transitive and strongly total, and that verifies $C(S)=G(S, Q)$ for any $S \in \mathcal{B}$.

\subsection{Consistency in fuzzy choice: some implications}

We proceed to study possible connections between the consistency axioms that we have stated in Subsection 3.1 .

It will become apparent that conditions $\mathrm{H} 1$ an $\mathrm{H} 2$ are more suitable to establish comparisons than WH1 and WH2.

Henceforth we assume $*$ the minimum t-norm, unless otherwise stated.

Observe that G-rationality trivially implies FB. Since the Fuzzy Arrow Axiom implies G-rationality in the presence of $\mathrm{H} 1$ and $\mathrm{H} 2$ as recalled above, it is obvious that under such restrictions FAA implies FB.

Lemma 1 below proves that the Fuzzy Arrow Axiom implies the fuzzy Chernoff property irrespective of the structure of $\mathcal{B}$, both in our restrictive sense and in Georgescu's approach:

Lemma 1 If a fuzzy choice function $C$ satisfies $F A A$ then it also satisfies $F C H$, hence $F C H^{*}$.

Despite this positive result, as announced above our dual version of FCH is not implied by FAA even when $\mathrm{H} 1$ and $\mathrm{H} 2$ are imposed:

Counterexample 1 The FAA does not imply the fuzzy dual Chernoff condition (FDCH) even under conditions $H 1$ and H2. Consider the set $X=\{x, y\}$ and $S$ the fuzzy subset of $X$ satisfying $S(x)=0.5$ and $S(y)=1$. Take $\mathcal{B}=\{X, S,\{x\},\{y\}\}$ and the choice function defined as follows. The choice func-

\begin{tabular}{|c|c|c|c|c|c|c|c|c|}
\hline & $X$ & $S$ & $x$ & $y$ & $C(X)$ & $C(S)$ & $C(x)$ & $C(y)$ \\
\hline$x$ & 1 & 0.5 & 1 & 0 & 1 & 0.5 & 1 & 0 \\
\hline$y$ & 1 & 1 & 0 & 1 & 0.5 & 1 & 0 & 1 \\
\hline
\end{tabular}

Table 1: Choice function corresponding to Counterexample 1. Brackets have been omitted.

tion $C$ satisfies $H 1, H 2$ and the FAA but $I(S, X)=$ 1 and $I(C(S), C(X) \cap S)=0.5$.

Counterexamples 2 and 3 below prove that even if conditions WH1 and WH2 are imposed the Fuzzy Arrow Axiom implies neither the fuzzy Superset property nor the fuzzy Property $\gamma$.

Counterexample 2 The Fuzzy Arrow Axiom does not imply the fuzzy superset property under conditions WH1 and WH2. Consider the set $X=\{x, y, z\}$ and $\mathcal{B}=\{X, T, S,\{x, y\},\{x, z\},\{y, z\},\{x\},\{y\},\{z\}\}$, where $T$ and $S$ are defined in Table 2. Consider the fuzzy choice function $C$ also defined in Table 2. This choice function satisfies the Fuzzy Arrow Axiom. Conditions WH1 and WH2 are also satisfied, but $C$ does not satisfy the fuzzy Superset property: $I(S, T)=1$ and $I(C(T), C(S))=1$ but $E(C(T), C(S))=I(C(S), C(T))=0.7$. 


\begin{tabular}{|c|c|c|c|c|c|c|c|c|c|}
\hline & $X$ & $T$ & $S$ & $x, y$ & $x, z$ & $y, z$ & $x$ & $y$ & $z$ \\
\hline$x$ & 1 & 0.9 & 0.9 & 1 & 1 & 0 & 1 & 0 & 0 \\
\hline$y$ & 1 & 0.7 & 0.6 & 1 & 0 & 1 & 0 & 1 & 0 \\
\hline$z$ & 1 & 0.7 & 0.7 & 0 & 1 & 1 & 0 & 0 & 1 \\
\hline
\end{tabular}

\begin{tabular}{|c|c|c|c|c|c|c|}
\hline & $C(X)$ & $C(T)$ & $C(S)$ & $C(x, y)$ & $C(x, z)$ & $C(y, z)$ \\
\hline$x$ & 0.7 & 0.7 & 0.9 & 1 & 0.7 & 0 \\
\hline$y$ & 0.6 & 0.6 & 0.6 & 0.6 & 0 & 0.6 \\
\hline$z$ & 1 & 0.7 & 0.7 & 0 & 1 & 1 \\
\hline
\end{tabular}

Table 2: Choice function corresponding to Counterexample 2. Brackets have been omitted.

\begin{tabular}{|c|c|c|c|c|c|c|c|c|c|c|}
\hline & $X$ & $T$ & $S_{1}$ & $S_{2}$ & $x, y$ & $x, z$ & $y, z$ & $x$ & $y$ & $z$ \\
\hline$x$ & 1 & 0.9 & 0.9 & 0.6 & 1 & 1 & 0 & 1 & 0 & 0 \\
\hline$y$ & 1 & 0.9 & 0.6 & 0.9 & 1 & 0 & 1 & 0 & 1 & 0 \\
\hline$z$ & 1 & 0.5 & 0.5 & 0.5 & 0 & 1 & 1 & 0 & 0 & 1 \\
\hline
\end{tabular}

\begin{tabular}{|c|c|c|c|c|c|c|c|}
\hline & $C(X)$ & $C(T)$ & $C\left(S_{1}\right)$ & $C\left(S_{2}\right)$ & $C(x, y)$ & $C(x, z)$ & $C(y, z)$ \\
\hline$x$ & 0.5 & 0.5 & 0.9 & 0.6 & 0.6 & 0.5 & 0 \\
\hline$y$ & 0.5 & 0.5 & 0.6 & 0.9 & 1 & 0 & 0.5 \\
\hline$z$ & 1 & 0.5 & 0.5 & 0.5 & 0 & 1 & 1 \\
\hline
\end{tabular}

Table 3: Choice function corresponding to Counterexample 3. Brackets have been omitted.

Counterexample 3 The Fuzzy Arrow Axiom does not guarantee the fuzzy Concordance property under conditions WH1 and WH2. As a consequence it does not guarantee the fuzzy Property $\gamma$ either.

To prove it, consider the element $x$ in the situation defined by Table 3. It suffices to check that

$$
\begin{gathered}
C\left(S_{1}\right)(x) * C\left(S_{2}\right)(x)=\min (0.9,0.6)=0.6> \\
>0.5=C(T)(x)
\end{gathered}
$$

in order to observe $C\left(S_{1}\right) * C\left(S_{2}\right) \nsubseteq C(T)$.

In order to assess to which extent the Fuzzy Arrow Axiom is related to the fuzzy Property $\gamma$ (resp., the fuzzy Superset property), Counterexample 2 (resp., Counterexample 3) should be contrasted with Lemma 2 (resp., Lemma 3) below.

Lemma 2 Let $C$ be a fuzzy choice function defined on a domain $\mathcal{B}$. If $C$ satisfies the Fuzzy Arrow Axiom and conditions $H 1$ and $H 2$ are verified, then it satisfies the fuzzy Superset property.

Lemma 3 Let $C$ be a fuzzy choice function defined on a domain $\mathcal{B}$. If $C$ satisfies the Fuzzy Arrow Axiom and conditions $H 1$ and $H 2$ are verified, then it satisfies the fuzzy Property $\gamma$.

Only one extended implication from Figure 1 does not hold true when conditions $\mathrm{H} 1$ and $\mathrm{H} 2$ are imposed in the fuzzy context: the fuzzy Property $\gamma$ does not imply the fuzzy Binariness property. The following counterexample proves this assertion:

Counterexample 4 Consider the set $X=\{x, y\}$ and $\mathcal{B}=\{X, S,\{x\},\{y\}\}$ where $S$ is defined as in Table 4. The choice function considered in that table satisfies the fuzzy Property $\gamma$, but not the fuzzy
Binariness property. Observe that $C(S)(x)=0.5$, but

$$
S(x) * \wedge_{y \in X}(S(y) \rightarrow C(\{x, y\})(x))=0.9 .
$$

\begin{tabular}{|c|c|c|c|c|c|c|c|c|}
\hline & $X$ & $S$ & $x$ & $y$ & $C(X)$ & $C(S)$ & $C(x)$ & $C(y)$ \\
\hline$x$ & 1 & 0.9 & 1 & 0 & 1 & 0.5 & 1 & 0 \\
\hline$y$ & 1 & 1 & 0 & 1 & 1 & 1 & 0 & 1 \\
\hline
\end{tabular}

Table 4: Choice function corresponding to Counterexample 4. Brackets have been omitted.

The results proven in this section show that conditions WH1 and WH2 are too weak if we want to extend to the fuzzy context the connections between the consistency conditions presented previously that we had in the crisp case. Conditions H1 and H2 are far more convenient to this purpose.

\section{Sequential Choice and Consistency}

In abstract choice theory there is growing attention to non-standard decision mechanisms, especially the case where the decision maker proceeds by sequential application of rational (in some classical sense) criteria. Manzini and Mariotti [7] explain that it permits to give support to experimental evidence from psychology and marketing studies that contradicts the standard maximizing paradigm. They provide a simple example where a cyclical pattern of choice is explained by an appealing sequential procedure in social choice proposed by Tadenuma [11]. Since cycles of choice are forbidden in the model by maximization of well-behaved preferences, but appear in experiments on a regular basis, sequential choice permits to incorporate such observations into the axiomatic analysis. The question arises: What choices can be explained by the sequential application of rational choices? This is an issue where other relevant studies include García-Sanz and Alcantud [5] (for multivalued choice) and the references therein.

Needless to say, in order to study sequentially rational choice the normative implications of such mechanism must be investigated. The same is true in the fuzzy variation of the model.

In view of this motivation we now proceed to introduce the notion of sequential fuzzy choice as follows:

Definition 9 Let $X$ be a set of alternatives and $\mathcal{B}$ the set of all non-zero fuzzy subsets of $X$. Let $C_{1}, C_{2}$ be fuzzy choice functions defined on $(X, \mathcal{B})$. Then $C_{1} \circ C_{2}: \mathcal{B} \rightarrow \mathcal{F}(X)$ such that for each $S \in \mathcal{B}$, $\left(C_{1} \circ C_{2}\right)(S)=C_{1}\left(C_{2}(S)\right) \subseteq S$ is the sequential application of $C_{1}$ and $C_{2}$, also called the sequential fuzzy choice function $C_{1} \circ C_{2}$.

Similarly one can define the sequential application of the fuzzy choice functions $C_{1}, \ldots, C_{n}$. 
For simplicity we have defined sequential application of fuzzy choice functions defined on the whole collection of non-zero fuzzy subsets of $X$. Nevertheless the sequential application of $C_{1}$ and $C_{2}$ can be performed on domains other than this universal class. For example, let $F[X]$ be the collection of non-zero fuzzy subsets of any crisp finite subset of $X$, i.e., $F[X]=\{A \subseteq f[X]: A$ is non-zero $\}$. Then when $C_{1}$ is defined on either $(X, F[X])$ or $(X, f[X])$ and $C_{2}$ is defined on $(X, F[X])$ the sequential application of $C_{1}$ and $C_{2}$ is well-defined too.

Remark 2 Consider a set of alternatives $X$ and let $\mathcal{B}$ be a subset of $\mathcal{F}(X) \backslash \emptyset$. Let us recall that given two sets $S$ and $T$ in $\mathcal{B}$, if there is $x \in X$ such that $S(x)>T(x)=\alpha$ then $I(S, T) \leq \alpha$ and as a consequence, $E(S, T) \leq \alpha$.

Remark 3 Consider a finite set of alternatives $X$ and let $\mathcal{B}$ be a subset of $\mathcal{F}(X) \backslash \emptyset$. If $I(S, T)=$ $\alpha$ for some $\alpha<1$, then there exists at least one element $x \in X$ such that $S(x)>\alpha=T(x)$. As a consequence, if $E(S, T)=\alpha$, then at least one of the following two situations hold:

- there exists one element $x$ such that $S(x)>$ $\alpha=T(x)$,

- there exists one element y such that $T(y)>$ $\alpha=S(y)$.

Theorem 1 Let $X$ be a finite set of alternatives and $\mathcal{B}$ a non-empty subset of $\mathcal{F} \backslash\{\emptyset\}$. Let $C_{1}, \ldots, C_{n}$ be a finite set of fuzzy choice functions defined on $(X, \mathcal{B})$. If $C_{1}, \ldots, C_{n}$ satisfy the Fuzzy Arrow Axiom and the sequential fuzzy choice function $C_{1} \circ \cdots \circ C_{n}$ is well-defined then $C_{1} \circ \cdots \circ C_{n}$ also satisfies the Fuzzy Arrow Axiom too.

Georgescu [6, Theorem 6.38] establishes the equivalence between FAA and the property that the choice function is full-rational, under conditions $\mathrm{H} 1$ and $\mathrm{H} 2$ and for the minimum t-norm. This crucial result permits to take advantage of Theorem 1 in order to deduce that the sequential application of full-rational fuzzy choice functions is full-rational, i.e.:

Corollary 1 Let $X$ be a finite set of alternatives and $\mathcal{B}$ a non-empty subset of $\mathcal{F} \backslash\{\emptyset\}$. Let $C_{1}, \ldots, C_{n}$ be a finite set of fuzzy choice functions defined on $(X, \mathcal{B})$ satisfying conditions $H 1$ and $H_{2}$.

If $C_{1}, \ldots, C_{n}$ are full-rational then $C_{1} \circ \cdots \circ C_{n}$ is full-rational too.

Corollary 1 is proven indirectly by the recourse to Georgescu [6, Theorem 6.38] coupled with Theorem 1. However the researcher may need to avail herself of an explicit procedure for actually rationalizing the sequential application of rational fuzzy choice functions. We now provide a solution to this issue under the only requirement of G-rationality:
Corollary 2 Let us fix a set of alternatives $X$ and $\mathcal{B}$ a non-empty subset of $\mathcal{F} \backslash\{\emptyset\}$ satisfying condition H2. Given two G-rational fuzzy choice functions $C_{1}$ and $C_{2}$ with associated weak preference relations $R_{1}$ and $R_{2}$, if $C_{2} \circ C_{1}$ is $G$-rational then the fuzzy preference relation associated with the composition of $C_{1}$ and $C_{2}, C_{2} \circ C_{1}$, is the relation

$$
R^{12}(x, y)=R_{1}(x, y) *\left(R_{1}(y, x) \rightarrow R_{2}(x, y)\right) .
$$

Notice that the relation $R^{12}$ associated with the composition in Corollary 2 is a fuzzy version of a lexicographic relation. Thus our explicit solution has an intuitive natural interpretation. We emphasize the fact that it does not require any additional restriction on the type of rationality of the original fuzzy choice functions, whereas Theorem 1 refers to the full-rational case.

Remark 4 Georgescu [6, Definition 5.38] introduces the concept of G-normal fuzzy choice function. In principle it establishes a particular form of G-rationality where the fulfilment of the optimality condition must hold true for the fuzzy revealed preference $R$ ([6, Definition 5.16]). But because $G$ rationality is equivalent to G-normality under the minimum t-norm ([6, Proposition 5.40]) we trivially derive: in the conditions of Corollary 2, $C_{2} \circ C_{1}$ is $G$-normal; and $R^{12}$ is the revealed preference associated with $C_{2} \circ C_{1}$.

\section{Acknowledgements}

The first author is grateful to M. D. García-Sanz and Rocío de Andrés Calle for valuable discussions. The first author acknowledges financial support by the Spanish Ministerio de Economía y Competitividad (Project ECO2012-31933).

\section{Conclusion}

The new concept of sequential application of fuzzy choice functions may be a tool to escape paradoxical observed behaviors. For example, we have found that there are fuzzy choice functions that do not verify $\mathrm{FCH}$ but can be explained as the sequential application of two G-rational fuzzy choice functions. We have settled the basis for future investigations of this idea. Many developments could be made in this respect. For example, the exploration of which consistency axioms ensure a consistent behavior at the end of the sequential process, in line with Theorem 1. The interplay with other rationalizability ideas like M-rationality is another possibility for research.

\section{References}

[1] K. Arrow, Rational choice functions and orderings, Economica, 26:121-127, 1959. 
[2] A. Banerjee, Fuzzy choice functions, revealed preference and rationality, Fuzzy Sets and Systems, 70:31-43, 1995.

[3] R. Belohlavec. Fuzzy Relational Systems: Foundations and Principles, Kluwer Academic Publishers, 2002.

[4] J. Fodor, M. Roubens. Fuzzy Preference Modelling and Multicriteria Decision Support. Kluwer Academic Publishers, Dordrecht (1994).

[5] M. D. García-Sanz and J. C. R. Alcantud, Sequential rationalization of multivalued choice, Mathematical Social Sciences, 74:29-33, 2015.

[6] I. Georgescu. Fuzzy Choice Functions: A Revealed Preference Approach, 2007.

[7] P. Manzini and M. Mariotti, Sequentially rationalizable choice, American Economic Review, 97:1824-1839, 2007.

[8] D. Martinetti, S. Montes, S. Díaz and B. De Baets, On Arrow-Sen style equivalences between rationality conditions for fuzzy choice functions, Fuzzy Optimization and Decision Making, 13: 369-396, 2014.

[9] K. O. May, Intransitivity, utility, and the aggregation of preference patterns, Econometrica, 22:1-13, 1954.

[10] A. K. Sen, Choice functions and revealed preference, Review of Economic Studies, 38:307317, 1971.

[11] K. Tadenuma, Efficiency first or equity first? Two principles and rationality of social choice, Journal of Economic Theory, 104:462-72, 2002.

[12] A. Tversky, Intransitivity of preferences, Psychological Review, 76:31-48, 1969.

[13] H. Uzawa, Note on preference and axioms of choice, Annals of the Institute of Statistical Mathematics, 8:35-40, 1956. 